\title{
Activos y estructuras de oportunidades de movilidad. Una propuesta analítica para el estudio de la accesibilidad por transporte público, el bienestar y la equidad
}

Diego Hernández. Universidad Católica del Uruguay, Montevideo, Uruguay.

RESUMEN | Uno de los desafíos actuales para los estudios sobre movilidad y transporte en América Latina es adoptar un enfoque multidimensional de la movilidad que trascienda la noción más estrecha de desplazamiento o viaje y que, a la vez, permita integrar este tema a la discusión más general acerca del efecto de la movilidad sobre la pobreza y la exclusión social. Este artículo aborda el desafío y propone un marco analítico que recoja la —aún incipiente en nuestra región- experiencia teórico-empírica sobre estos fenómenos. Luego de revisar la literatura y argumentar a favor de la adopción de diversos postulados, se propone el enfoque de activos y estructuras de oportunidades para el estudio de la accesibilidad. Esta es concebida como el grado de ajuste entre las estructuras de oportunidades de movilidad, y los recursos y activos con que cuentan los hogares para aprovecharlas. El esquema propuesto permite maximizar la comprensión del efecto de la movilidad sobre el bienestar y el rol del transporte público.

PALABRAS CLAVE | Movilidad, transporte urbano, desarrollo humano, vulnerabilidad.

ABSTRACT | One of the major current challenges for mobility and transport studies in Latin America is the adoption of a multidimensional approach that goes beyond the narrower notion of trip and that does not neglect a broader discussion about mobility's effect on poverty and social exclusion. This article seeks to tackle this challenge and posits an analytical framework that takes into account the theoretical and empirical regional background on these phenomena. After reviewing existing literature and affliating to some necessary assumptions, it proposes the assets and opportunities structures approach as a useful device to study accessibility. This concept is defined as the level of adjustment between the structures of opportunity for mobility and household's resource and assets to take advantage from them. I argue that this framework is adequate in order to maximize understanding of mobility's effect on welfare and the role of public transport.

KEY WORDS | Mobility, urban transportation, human development, vulnerability.

Recibido el 15 de septiembre de 2010, aprobado el 1 de octubre de 2011

E-mail: dohernan@uc.cl 


\section{Introducción}

Uno de los grandes desafíos a los que se enfrentan las ciudades contemporáneas es el de la movilidad cotidiana. En un contexto en el que para realizar varias actividades se debe recorrer importantes distancias y, por ende, utilizar medios motorizados, la discusión acerca del efecto de la movilidad sobre la pobreza y la exclusión social es insoslayable. Nuestra región no solo no escapa a esta problemática, sino que podría ser un ejemplo de alguna de sus manifestaciones más severas. Por todo esto, es más que deseable la reflexión sobre las herramientas teóricas que permitan desarrollar conocimiento en materia de movilidad y su relación con el bienestar de las personas que habitan en las ciudades; y, más específicamente, sobre el rol que cumple el transporte público en dicha ecuación. Ese es justamente el objetivo de este trabajo: proponer un marco analítico que recoja la —aún incipiente en nuestra región- experiencia teórico-empírica sobre estos fenómenos.

En tal sentido, luego de revisar la literatura y argumentar en torno a algunos de los postulados que hoy sustentan la discusión sobre esta materia, se propone la adopción del enfoque de activos y estructuras de oportunidades para el estudio de la accesibilidad. Esta es concebida como el grado de ajuste entre las estructuras de oportunidades de movilidad, y los recursos y activos con los que cuentan los hogares para aprovecharlas. Como se argumentará a lo largo del artículo, este modelo reúne varios rasgos que lo hacen deseable. En primer lugar, permite dar cuenta de la "doble" condición de la movilidad, un activo con que cuentan los hogares - cuya distribución depende de las oportunidades y de los recursos de los hogares - y que, al mismo tiempo, permite acceder a otros bienes. Por otra parte, posibilita la inclusión, desde una perspectiva global, del transporte público en tanto política de la arena social cuyo desempeño impactará en el bienestar de las personas. Adicionalmente, se trata de un esquema flexible e integral mediante el cual es posible considerar variables individuales del hogar y factores estructurales que no están bajo su control.

El artículo comienza situando y definiendo la noción a partir de la cual elaborará conceptualmente la accesibilidad. Una vez que se haya recorrido y precisado ese concepto, se discutirán sus implicancias en términos del bienestar de las personas y en cuanto a las rutas por las cuales la literatura existente ha establecido ese vínculo. Toda esta reflexión dejará bases firmes para discutir un modelo que pueda abordar todas las dimensiones referidas, pero sin perder de vista el acceso al bienestar. En ese punto, el artículo se concentrará en la presentación de los fundamentos del enfoque de activos, vulnerabilidad y estructura de oportunidades (AVEO) y los aportes que supondría su adopción para el estudio de la accesibilidad. Es en este marco, además, donde se realizarán algunas consideraciones conceptuales acerca de la naturaleza del transporte público en el contexto urbano, que vienen a complementar la adecuación de este enfoque. 


\section{Accesibilidad y bienestar}

\section{El concepto de accesibilidad}

La definición de accesibilidad le otorga un rol relevante a nociones tales como derecho, ejercicio de la ciudadanía o inclusión. En última instancia, lo que se destaca es la naturaleza pública de la movilidad y la accesibilidad, reconociéndose el hecho de que se trata de un bien que debería ser protegido por el conjunto de la sociedad. En términos de Ascher (2005), "hoy la movilidad es una condición clave de acceso al mercado laboral, a una vivienda, a la educación, a la cultura y el ocio, a la familia. El derecho a trabajar, a tener una vivienda, a recibir capacitación, ahora implica el derecho a la movilidad (...) en cierto sentido este derecho a la movilidad es una precondición de los otros derechos" (p. 19). Por supuesto que esta aparente declaración de carácter político tiene un correlato conceptual muy fuerte. Da a entender la existencia de una relación prácticamente causal entre la movilidad y el acceso a otros bienes y oportunidades con impacto directo en la calidad de vida de las personas.

El debate teórico fundamental en el que se inscriben estas reflexiones proviene de las ciencias sociales y advierte sobre la existencia de una interrelación entre la movilidad territorial y la movilidad social. Admite un rol significativo de la movilidad territorial o espacial en la producción y reproducción de las estructuras sociales, en este caso en relación con el bienestar social. ${ }^{1}$ La movilidad y la accesibilidad constituyen un vector más de la desigualdad existente en las ciudades de nuestro continente, tal como lo son los procesos de segregación urbana o el acceso a la educación. Como se verá más adelante, el fenómeno de la movilidad cotidiana, y la accesibilidad en particular, han sido menos estudiados en el continente. No obstante ello, existen indicios que hacen plausible afirmar que la movilidad territorial es una condición de la movilidad social (ascendente o descendente) (Gutiérrez 2009b, p. 2).

Miralles y Cebollada (2003) afirman que la accesibilidad "hace referencia a la facilidad con que cada persona puede superar la distancia que separa dos lugares y de esta forma ejercer su derecho como ciudadano (...) la accesibilidad, además de una dimensión territorial, también es una característica individual con relación al número de opciones que tienen los diferentes ciudadanos para acceder a los lugares y a las actividades" (p. 14). Esta definición va al núcleo central de la discusión: la capacidad de las personas de acceder a los lugares donde se brindan algunos servicios y oportunidades necesitados - o deseados - por las personas. Como se deriva de estas definiciones, uno de los componentes fundamentales tiene que ver con la noción de capacidad para "llegar a". Esto implica subrayar la noción de potencialidad, vale decir, que dicha capacidad no se asocia a lo que el individuo sí logra hacer, sino que es un atributo cuyo estudio requiere ir más allá de los movimientos conocidos u observables.

1 Entendiendo "bienestar" como aquel conjunto de bienes básicos necesarios para el desarrollo de la persona y el acceso a canales de integración y movilidad social ascendente (en términos agregados, referiría al desarrollo social de una sociedad). Entre ellos se cuentan bienes vitales, tales como la alimentación, salud, educación, vivienda, cuidados en escenarios de vulnerabilidad (niñez y vejez). También podrían considerarse bienes "posmaterialistas", tales como ocio y esparcimiento y el acceso a bienes culturales. 
Vale destacar que un individuo puede contar con muy poca capacidad de movilizarse a grandes distancias en el espacio y, sin embargo, tener muy buena accesibilidad por cercanía. Y a la inversa, puede disponer de mucha facilidad de movimiento hacia muchos puntos de la ciudad, pero no hacia el que necesita llegar, por lo que, a pesar de su alto nivel de movilidad, su accesibilidad sigue siendo baja. En tal sentido, la localización de las actividades desempeña un papel relevante, en el sentido de que, en teoría, la superación de las distancias puede darse desde el individuo desplazándose a las actividades o desde las actividades "desplazándose" hacia ellos (localización cercana). Así, por ejemplo, los problemas de accesibilidad al trabajo se podrían resolver de dos formas: con un adecuado sistema de transporte que lleve a las personas a las zonas con alta concentración de oportunidades laborales, o por medio del emplazamiento de oportunidades cercanas al lugar de residencia.

\section{Accesibilidad, bienestar y equidad}

Como se mencionó antes, ya desde su definición el concepto de accesibilidad sugiere una vinculación estrecha con nociones como bienestar y equidad. En general, la literatura respectiva parte de un abordaje similar al aquí propuesto, en tanto concibe la movilidad como un bien que permite acceder a otros bienes, e indaga en la forma en que estos procesos tienen lugar y en cómo afectan a los hogares e individuos según sea, por ejemplo, su nivel socioeconómico. Concretamente, se pregunta qué sucede con la movilidad y la accesibilidad de las personas - especialmente los sectores de bajos ingresos - y advierte acerca del impacto que tiene sobre ellas la capacidad de superar las distancias geográficas entre un punto y otro para poder participar de la vida en la ciudad y beneficiarse de las oportunidades y recursos que el espacio urbano ofrece.

En efecto, esta relación ha sido puesta a prueba y descrita de forma relativamente frecuente, en especial por la literatura proveniente de países industrializados. Una de las manifestaciones de esta producción es la línea de estudios sobre la hipótesis del spatial mismatch, la que postula que las oportunidades laborales para los sectores de bajos ingresos se localizan lejos de sus lugares de residencia. En Estados Unidos, esta hipótesis fue de particular importancia para analizar la inserción deficitaria de la población negra de las áreas centrales de la ciudad en las mejores oportunidades localizadas en los suburbios (Blumenberg, 2004; Gobillon, Selod \& Zenou, 2007; Kain, 1968, 1992). Su énfasis está puesto en el desfase geográfico entre el lugar de residencia de los menos favorecidos y sus oportunidades laborales, y se concentra menos en los mecanismos que determinan los obstáculos a la movilidad.

Es posible identificar otra línea algo más centrada en el fenómeno de la movilidad y accesibilidad. Una de las primeras constataciones de los análisis correspondientes es que las cuestiones relacionadas a la movilidad y la accesibilidad no constituyen un tema de libre elección personal y pueden presentar muy fuertes constreñimientos estructurales (Massot \& Orfeuil, 2005; Wenglenski \& Orfeuil, 2006). Es importante, pues, no caer en la "tentación" de pensar que la movilidad lle- 
ga a todos los estratos sociales por igual. En este sentido, Kaufmann (2002) plantea justamente que no se debe confundir entre mayor velocidad de transporte y movimiento como un imperativo de las sociedades modernas, con que efectivamente ese sea un atributo de toda la población. En pocas palabras, el punto de partida para pensar en movilidad, accesibilidad y bienestar es que estas condiciones no pueden darse por establecidas, y que constituyen un atributo que no se distribuye de forma equitativa entre las personas y los hogares. Hay personas que tienen más posibilidades de movilizarse que otras, hay quienes pueden hacerlo mucho más rápido que otros, hay quienes pueden hacerlo en mayores direcciones que otros.

Esta literatura ha profundizado de forma más específica en el análisis de la accesibilidad y su interacción con otros rasgos socioeconómicos. Así, es posible encontrar investigaciones que dan cuenta de la pauta regresiva con que se distribuye la accesibilidad entre los distintos estratos sociales, tanto para la obtención de bienes más clásicos, como estudio, trabajo o compras (Cebollada \& Avellaneda, 2008; Miralles-Guasch, 2006; Social Exclusion Unit, 2003; Wenglenski, 2003; Wenglenski 2006; Wenglenski \& Orfeuil, 2006), como para otras actividades que trascienden la esfera de estas actividades "imperativas" (Cass, Shove \& Urry, 2005; Currie et al., 2009; Currie \& Stanley, 2008; Flamm, Jemelin \& Kauffmann, 2008; Larsen, Urry \& Axhausen, 2006; Neutens, Delafontaine \& Schwanen, 2010; Viry, Kaufmann \& Widmer, 2009).

Vale mencionar que uno de los componentes que sobresalen de esta literatura es la identificación de minorías excluidas de la movilidad. Es así que varios estudios hacen marcado hincapié en colectivos específicos, como los inmigrantes o los sectores ubicados en los estratos sociales más bajos. Asimismo, algunos de los factores de exclusión incluyen temas como competencias lingüísticas, que dan la pauta de una exclusión preexistente y bastante extendida. En el caso de América Latina, el estudio de la accesibilidad y la movilidad trasciende la atención a minorías excluidas, en tanto la presencia de situaciones de pobreza y factores asociados a ella es mucho más masiva. Lo mismo podría decirse de la dependencia de amplios estratos de la población respecto del transporte público, situación que continúa siendo masiva en nuestro continente (Corporación Andina de Fomento, 2009a, 2009b).

Como ya se mencionó, los estudios de la accesibilidad en clave de bienestar y equidad son realmente escasos en nuestro continente. No obstante, es posible encontrar varias investigaciones que abordan el tema de forma más o menos directa. En el marco de estudios de pobreza, se sugiere la escasa accesibilidad como un factor más de agravamiento de la exclusión. De todas maneras, en este tipo de estudios es posible encontrar algunos que ponen algo más de énfasis en la cuestión de la movilidad. En general lo hacen con relación al empleo y poniendo más atención en el spatial mismatch y, eventualmente, en sus causas. Sus fuentes de información consisten en censos de población o encuestas periódicas producidas en el marco de los sistemas estadísticos nacionales (Antico, 2005; Aranha, 2005; Brito \& De Souza, 2005; Caiado, 2005; Kaztman, 2009; Rodríguez Vignoli, 2008).

En general, los estudios más específicos tienen un acentuado énfasis cualitativo y abordan dimensiones similares a los reseñados anteriormente (Avellaneda 
García, 2007; Cebollada \& Avellaneda, 2008; Gutiérrez, 2009b; Jaramillo, 1993; Jirón, 2008; Lazo, 2008). Además de estos estudios, es posible encontrar en algunas ciudades latinoamericanas la difusión de estudios basados en las encuestas origen-destino que describen aspectos básicos de movilidad observada, partición modal, etcétera. En general, estos documentos provienen de las propias entidades gubernamentales a cargo de la supervisión del transporte público. Lo que resulta evidente en América Latina es que no existen por el momento discusiones acabadas sobre medidas sistemáticas de accesibilidad y que, sin ser reduccionistas, den pautas de clasificación de las zonas de la ciudad algo más amplias (Hernández \& Witter, 2011). La producción, aunque escasa, es categórica en resaltar el papel central que desempeña el aspecto tarifario y económico en la accesibilidad por transporte público de los sectores populares. En efecto, los estudios de la región señalan la escasa disponibilidad de medios económicos para pagar la tarifa del transporte público como el eje estructurador de las dificultades de accesibilidad. Como se mencionó más arriba, no aparece la noción de grupo excluido absolutamente del sistema debido a, por ejemplo, no tener auto, sino la de grupos sociales cuyo acceso a los servicios disponibles puede resultarles prohibitivo o cuyas alternativas en términos de movilidad son de baja calidad o de muy alto costo personal.

\section{Dimensiones relevantes y obstáculos a la accesibilidad}

Varios estudios identifican los elementos que pueden obstaculizar o facilitar la accesibilidad de las personas, los que constituyen en cierta medida sus determinantes. Más allá de las distintas variantes y planteos existentes en la literatura (Cass et al., 2005; Church, Frost \& Sullivan, 2000; Flamm \& Kaufmann 2006; Kaufmann, Bergman \& Joye, 2004; Kaufmann et al., 2009; Social Exclusion Unit, 2003; Titheridge, 2006; Urry, 2007) y centrando la discusión en la accesibilidad por transporte público, es posible identificar cuatro dimensiones que deberían ser consideradas:

a) De oferta: relacionada al bien que se provee, a la red ofrecida y su extensión, los tiempos que impone a quien traslada, sus rasgos (horarios, regularidad, extensión temporal), su calidad en términos de la comodidad y seguridad de su material tanto rodante como estático, y la información que pone a disposición de los individuos.

b) De institucionalidad: refiere a los componentes que definen el grado de desmercantilización monetaria de esa red. Entre ellos se incluye la estructura tarifaria y de regulación, la introducción de subsidios y la definición de aquellos sobre quienes recae, y el diseño de las reglas de uso con relación al pago de la tarifa, además de aspectos puramente organizativos, tales como operadores y estructura de propiedad.

c) De rasgos individuales: referente a atributos de las personas y los hogares en cuanto a ingresos, el tiempo disponible y la forma de organizarlo (relacionado a sus requerimientos de actividades), y las habilidades y destrezas para dominar el sistema y su funcionamiento y poder acceder físicamente a él. 
d) De forma urbana: relacionada a dinámicas socioterritoriales que responden en mayor medida a factores estructurales que a decisiones individuales, como la localización de las actividades y residencia de los distintos estratos socioeconómicos.

Hasta aquí se han expuesto los fundamentos básicos relacionados con el concepto de accesibilidad, así como una síntesis de las principales dimensiones que emergen como potenciales obstáculos a la accesibilidad. La pregunta que resta abordar es, entonces, cómo manejar todos estos elementos dentro de un esquema analítico común que contemple la interacción entre ellos. También es necesario albergar la interacción entre la accesibilidad y las restantes dimensiones del bienestar, considerando en qué medida unas pueden convertirse en impulsos o frenos de las otras, y viceversa.

El concepto de motilidad - diferente terminológicamente, pero muy similar en su contenido al de accesibilidad- es una primera aproximación para este rompecabezas. Kaufmann et al. (2004) definen la motilidad como "la capacidad de ser móvil en el espacio social y geográfico o como la manera en que las entidades, de acuerdo con sus circunstancias, acceden y se apropian de la capacidad de movilidad socioespacial" (p. 750). Para estos autores, la motilidad, este potencial de movilidad, se transforma en un capital de las personas. A la vez, dicho capital va a estar íntimamente relacionado a la posibilidad de contar con otros capitales y se distribuye de forma desigual a lo largo de los distintos estratos.

Este es uno de los componentes centrales de la propuesta desarrollada aquí: la noción de capitales o recursos movilizados por parte de las personas y los hogares, así como la interdependencia de esos capitales o recursos para su proceso de acumulación o, expresado de otra forma, el logro de niveles mínimos de bienestar. Valiéndose del esquema AVEO, esta propuesta avanza de forma más explícita en la discusión sobre la naturaleza de la accesibilidad como capital con que cuenta un hogar. También avanza en la forma de integrarlo a una discusión más general sobre bienestar y en la incorporación del control que ejercen los hogares sobre los recursos como una de las dimensiones relevantes para el análisis. Adicionalmente, el esquema AVEO permite discutir a dos niveles: por un lado, el rol de la accesibilidad en la acumulación de otros recursos; y por otro, la forma en que los recursos de accesibilidad se relacionan entre sí. A continuación se presentan los postulados generales del esquema y una discusión específica sobre accesibilidad.

\section{Activos, estructuras de oportunidades y riesgos de movilidad: un marco analítico sintético}

El enfoque AVEO (activos, vulnerabilidad y estructura de oportunidades) surge de la necesidad de conocer más sobre la heterogeneidad de la pobreza y la lógica de actuación de los hogares de menores ingresos o, en otras palabras, los activos con que cuentan esos hogares y la forma en que los "gestionan". Moser (1998) afirma que conociendo este portafolio a la vez que las estrategias de su utilización, es posible facilitar 
la intervención social para que los hogares pobres puedan hacerlo de forma "productiva”. Kaztman (1999) plantea además que es necesario atender en mayor medida a las estructuras de oportunidades de una sociedad, ya que el grado de vulnerabilidad de un hogar estaría dado por el desfase entre ellas y su portafolio de activos. Siguiendo a Kaztman, "el concepto de activos no alcanza una significación unívoca si no está referido a las estructuras de oportunidades que se generan desde el lado del mercado, la sociedad y el Estado. En otras palabras, se sostiene que el portafolio y la movilización de activos de los hogares vulnerables, punto central en la formulación moseriana, solo puede examinarse a la luz de las lógicas generales de producción y reproducción de activos, que no pueden ser reducidas a la lógica de las familias y sus estrategias" (p. 34).

Concretamente, este esquema propone la existencia, por un lado, de estructuras de oportunidades, que son definidas como probabilidades de acceso a bienes, a servicios o al desempeño de actividades. Estas oportunidades inciden sobre el bienestar de los hogares, porque permiten o facilitan a los miembros del hogar el uso de sus propios recursos (Kaztman, 1999). Para este autor, "la obtención de niveles socialmente aceptables de bienestar pasa por el logro de empleos de buena calidad, por lo que la secuencia en el acceso a distintas oportunidades de bienestar tiende a organizarse de modo de maximizar la probabilidad de que los miembros del hogar se incorporen a actividades valoradas por el mercado" (p. 21).

Vale destacar, entonces, una suerte de encadenamiento jerárquico de estructuras de oportunidades donde, en virtud de la organización económica de la sociedad, la estructura de las oportunidades laborales es innegablemente la más importante. Pero, al mismo tiempo, ella da cuenta de la existencia de otras estructuras de oportunidades para la obtención de otros bienes, que a su vez condicionan una inserción eficaz o mejor aprovechamiento de oportunidades por parte de los individuos. Las estructuras de oportunidades facilitan el uso más eficiente de los recursos que ya posee el hogar, que por un lado mejoran directamente el bienestar y por el otro crean condiciones favorables a un mejor uso de sus recursos. En el caso de la accesibilidad per se, una mejora implica un aumento en el bienestar, pero además también permitiría usar de forma más eficiente los recursos humanos del hogar (por ejemplo, más tiempo disponible para otras actividades o la posibilidad de oportunidades laborales de mayor calidad o a más cantidad de oportunidades de ese tipo). Por otra parte, estas estructuras proveen nuevos activos o regeneran aquellos ya agotados, por ejemplo la educación (o reconversión educativa), que constituye claramente un recurso controlado por el hogar.

Como fuentes de las oportunidades se tiene a las tres grandes instituciones del orden social: el mercado, el Estado y la sociedad. Kaztman (1999) rescata dos roles que convierten al Estado en una fuente de oportunidades de acceso al bienestar muy significativa: i) el rol regulador, en tanto el mercado opera sobre instituciones reguladas en mayor o menor medida por el Estado; y ii) el rol vinculante entre mercado y sociedad, al establecer vínculos entre los recursos que circulan entre las tres esferas (por ejemplo, mediante un subsidio al transporte) (pp. 24-25). 
El aprovechamiento de estas estructuras de oportunidades dependerá de los recursos y activos de los hogares. Los recursos son todos los bienes que controla un hogar, sean tangibles o intangibles, mientras que la idea de activo (se podría mencionar el término "capital" también) se refiere al subconjunto de esos recursos cuya movilización permite el aprovechamiento de las estructuras de oportunidades existentes en un momento, ya sea para elevar el nivel de bienestar o para mantenerlo ante situaciones que lo amenazan (Kaztman, 1999, pp. 31-32).

Como puede observarse, a partir de este enfoque se podría identificar una tensión entre los recursos bajo el control de los hogares y las estructuras de oportunidades que responden a constreñimientos del medio. Como afirman los autores de esta línea, considerar los activos sin tener en cuenta las estructuras de oportunidad desdibuja su significado, por lo que hay que trascender el análisis de las estrategias de movilización de activos y complementarlo con el estudio del acceso a las estructuras de oportunidades, precisando si este acceso sigue algún tipo de pauta de estratificación. Este contraste, ajuste o adaptación es el que define la vulnerabilidad de los hogares (Kaztman, 1999, 2008, 2009; Kaztman \& Filgueira, 2006).

La Figura 1 ilustra el enfoque recién expuesto. Su presentación gráfica expresa de forma relativamente literal el relato argumental del enfoque AVEO. Allí pueden observarse los tres órdenes sociales fuentes de oportunidades y sus formas de intervención o bienes más significativos. En el extremo inferior aparecen los recursos de los hogares, agrupados de acuerdo con la distinción clásica entre capital físico, capital humano y capital social. También se indica el grado de control sobre los elementos del medio, control que se debilita a medida que los individuos u hogares se acercan a las estructuras de oportunidades, ya que, al no depender estas de la voluntad de las personas, operan como factor estructural de constreñimiento a su accionar. Por otro lado, ese control por parte de los hogares aumenta a medida que se acercan a los recursos más básicos y, en ese sentido, la movilización de tales recursos depende de su agencia. Los recursos sobre los que el hogar ejerce cierto control constituyen estructuras de riesgo, ya que su conformación determinará la capacidad de sus miembros para aprovechar las oportunidades en determinado momento histórico. Esa estructura de riesgos se complementa con otros factores que no necesariamente constituyen recursos del hogar, como, por ejemplo, la fase del ciclo vital en que se encuentra o la localización de su vivienda.

¿Cuál sería el lugar ocupado por la accesibilidad en este esquema? En primer término, se la considera como un activo del hogar que es clave para aprovechar otras estructuras de oportunidades. Así como un individuo debe estar saludable para obtener un empleo, también debe tener la capacidad de llegar al lugar donde esa actividad se desarrolla. Su rol va a ser similar al de otros activos, al igual que la forma de obtenerlos, ya que también existen estructuras de oportunidades de salud, de educación, etcétera. Lo que cambia es que, en su constitución como capital atribuible al hogar y a sus miembros, la accesibilidad depende en mayor medida que los demás recursos de las estructuras sociales más alejadas al control del hogar. Es por este motivo que se ubica algo por encima del resto de los capitales en el continuo 
referido a esa dimensión. En otras palabras, la accesibilidad es un capital que, como los demás, depende de la acumulación conjunta con los demás, pero es distinguible del resto, esto es, no se subsume en la forma de capital físico, humano o social.

FIGURA 1 | Esquema de activos y estructuras de oportunidades, donde se incluye la accesibilidad como un activo

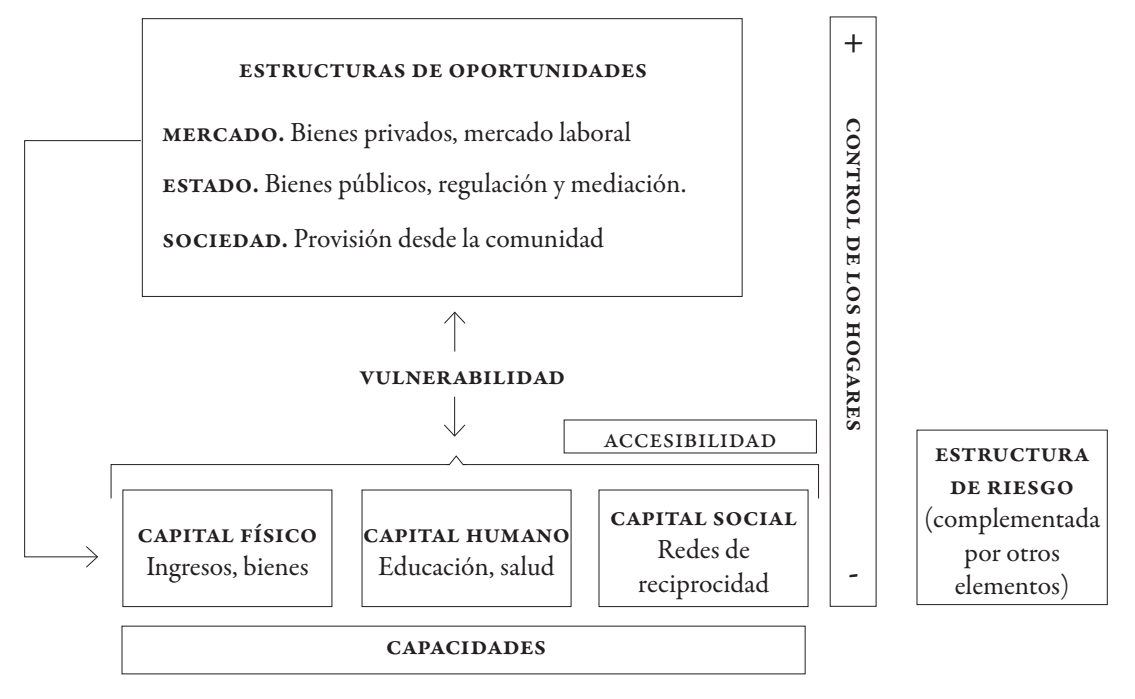

FUENTE ELABORACIÓN PROPIA.

Indudablemente, el caso de la accesibilidad y su naturaleza de recurso "intermedio" es central para entender el bienestar de los hogares. Debido al carácter de demanda derivada del transporte y la movilidad — no es un fin en sí mismo, sino que un medio para alcanzar otros-, este campo es un excelente ejemplo del encadenamiento de las múltiples combinaciones de oportunidades y recursos que se ajustan más o menos dependiendo de cada sociedad y cada hogar, y donde la obtención de determinados bienes puede ser vital para aprovechar otras estructuras de oportunidades, y así sucesivamente. Es entonces que, por un lado, puede colocarse la accesibilidad como activo o capital que es necesario movilizar para aprovechar estructuras de oportunidades de diversa índole (por ejemplo, laborales, de salud o de educación). Por el otro, la propia accesibilidad surge de los recursos en control de los hogares y de cuánto les permite aprovechar las oportunidades de movilidad existentes. El ajuste o desajuste entre estos dos componentes es lo que va a determinar el grado de vulnerabilidad en materia de accesibilidad. Y más concretamente, en el caso de los hogares de menores ingresos, este ajuste va a estar íntimamente ligado a las oportunidades que más se desmarquen del mercado. Se argumenta aquí que este conjunto de oportunidades se concentra en el transporte público, bien que se convierte entonces en el nudo central para el estudio de la accesibilidad. 
Uno de los atributos fundamentales que se desprenden de este esquema a la hora de discutir sobre accesibilidad, es su alto nivel de adecuación para sintetizar los muchos elementos operativos y conceptuales vigentes. Además, presenta la enorme ventaja de abarcar explícitamente de forma más completa que otros-y también sistémica - el conjunto del proceso de acceso al bienestar. Hay muchas estructuras de oportunidades y muchos tipos de recursos con los que un hogar cuenta. Entonces, cuando se piensa en la relación entre las estructuras de oportunidades y el portafolio de activos de los hogares, también se está pensando en el resto de los eslabonamientos para acceder a los distintos tipos de bienes, y este esquema hace muy parsimonioso - casi inevitable - ese recorrido.

Otra de las ventajas de este esquema consiste en que permite distinguir entre el recurso y el capital. Este último consiste en el conjunto de recursos cuya movilización permite concretamente el aprovechamiento de las estructuras de oportunidades. Es de esa combinación que resulta el nivel de vulnerabilidad de los hogares. Adoptar un esquema general como el que se presenta aquí facilita sustancialmente tener presente esa distinción y evita una visión lineal acerca de acumulación de recursos que no necesariamente se traducen en mayor bienestar para las personas. Una persona puede contar con determinados conocimientos que fueron útiles en algún momento histórico, pero que dejan de serlo en otro debido a, por ejemplo, avances tecnológicos que los vuelven obsoletos.

Este esquema responde, entonces, al extremo más alto de la jerarquía, a una visión del bienestar más global. No obstante ello, debido a su ductilidad, es posible posar una "lupa" en cada uno de sus componentes y descomponerlos en "pequeños esquemas AVEO" que den cuenta de cuáles son los recursos necesarios y las estructuras de oportunidades para cada uno de los capitales. A continuación se realiza ese ejercicio analítico para estudiar la accesibilidad. Una vez más, debe recordarse que este esquema es por definición uno que supone el eslabonamiento permanente de distintos componentes de una ruta al bienestar, y que esta ruta puede estar pautada por la inclusión o por la exclusión. En la Figura 2 se abre uno de esos eslabones, el de la accesibilidad. Allí se analizan cuáles son las estructuras de oportunidades y recursos cuyo grado de adaptación define la vulnerabilidad en materia de movilidad.

Siguiendo el postulado básico del esquema AVEO, se podría argumentar que la accesibilidad, como otros activos de los hogares, dependerá de las estructuras de oportunidades que el medio les ofrece y de sus capacidades para tomar ventaja de ellas. Un individuo puede estar rodeado de magníficas autopistas que no le signifiquen nada si no cuenta con un automóvil. De la misma forma, puede tener la red de transporte público muy cercana, pero ello tampoco significará demasiado si no puede pagar por usarla, no conoce cómo hacerlo o los tiempos en que lo traslada a los lugares donde se encuentran las oportunidades son demasiado extensos. Existen, entonces, diversas consideraciones respecto de cuáles son las oportunidades de accesibilidad que una ciudad ofrece, pero también cuáles son las capacidades de los 
individuos para aprovecharlas. Y el análisis de la accesibilidad - y del transporte público en particular - es uno de los eslabones primarios para conocer la vulnerabilidad con relación a otros bienes, ya que indica si la persona está en condiciones o no de superar las distancias geográficas entre su hogar y el lugar físico donde se proveen esos bienes.

FIGURA 2 | Esquema de activos y estructuras de oportunidades de accesibilidad

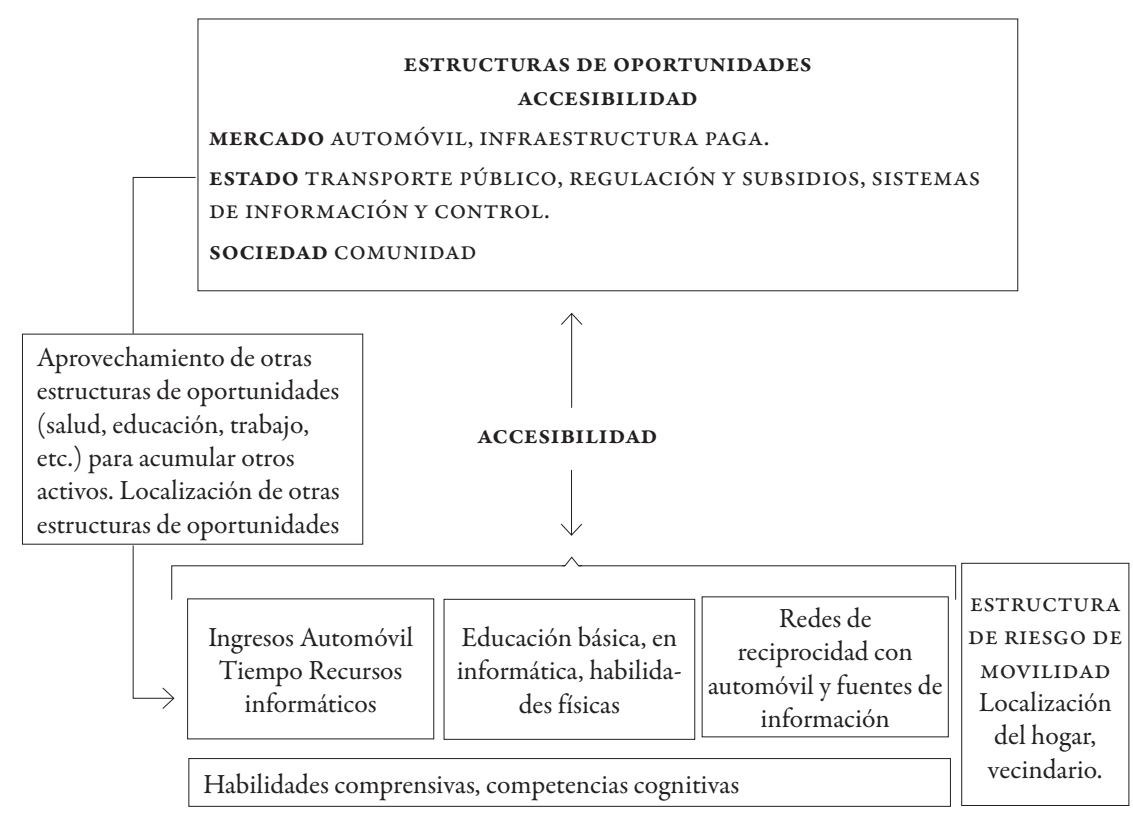

FUENTE ELABORACIÓN PROPIA.

Una de las particularidades de este esquema es que el nivel de accesibilidad tiene una línea de feedback que determina la provisión de nuevos recursos o recreación de los ya existentes. Dicha línea especifica que la acumulación de este capital está íntimamente relacionada a la posibilidad de acumular otros. Asimismo, en este esquema también aparece un factor adicional asociado a la acumulación de nuevos activos, cual es la forma urbana, que constituye una parte de los determinantes de la accesibilidad, aunque no en las estructuras de oportunidades o de los recursos básicos de los hogares. ${ }^{2}$ De todas maneras, la forma urbana determina en buena medida las distancias impuestas a los individuos, así como los medios a su alcance para recorrerlas. Esta variable hace que algunos recursos sean más o menos adecuados

2 La forma urbana está determinada en buena medida por las estructuras de oportunidades de otros bienes. Así por ejemplo, la localización de las oportunidades laborales tendrá que ver con la estructura de oportunidades en ese ámbito. Lo mismo se podría afirmar para otros sectores, como la salud o la educación. 
para aprovechar las estructuras de oportunidades de accesibilidad, en particular las que implican desplazamiento.

En cuanto a los recursos de los hogares, se los puede apreciar clasificados en los tres grandes grupos de capital: físico, humano y social. Básicamente son los mismos que para el esquema general porque, en última instancia, son aquellos recursos sobre los que el hogar tiene máximo control para su movilización.

Entre los recursos físicos se destacan en primer lugar los ingresos, que determinarán la capacidad de pago de medios de desplazamiento. También se contará la disponibilidad de opciones motorizadas privadas y otros activos de carácter informacional relacionados al acceso a información. Asimismo, entre los recursos físicos que se vuelven relevantes a los efectos de la accesibilidad está el tiempo con que cuenta la persona. Este, aunque intangible, se agota con su uso, y a la vez está pronunciadamente condicionado por su carácter indivisible y limitado para cada día.

Dentro del capital humano necesario para movilizar estructuras de oportunidades de accesibilidad, se contarán algunas credenciales básicas en materia de utilización de recursos virtuales, tanto para obtener información como para evitar desplazamientos innecesarios o minimizar el tiempo invertido en desplazamientos a partir de, por ejemplo, adecuarse a los horarios del transporte público. Por supuesto que también se contarán aspectos de formación básicos, que serán de menor o mayor complejidad. ${ }^{3}$ El otro recurso relevante en esta categoría tiene que ver con la capacidad física, recurso básico para movilizarse y hacer uso de los distintos medios, desde los activos - especialmente la caminata - hasta otros como el transporte público. Así, por ejemplo, de contarse con autobuses equipados para personas en sillas de ruedas, los recursos requeridos en materia de capital humano que permitan aprovechar esa oportunidad de movilidad serán menores.

Finalmente, el capital de carácter social es el que podría provenir de la participación en redes que derivaran en obtención de opciones motorizadas — por ejemplo, un familiar con automóvil- o en insumos de información o monetarios para la maximización de las oportunidades de movilidad.

La cartera de recursos de movilidad con que cuentan los hogares se complementa con un conjunto de competencias y habilidades que son funcionales a su movilización, y apuntan a la dimensión de apropiación y comprensión de las oportunidades y lo que estas ofrecen. Sumado a su localización y otros aspectos de forma urbana, configuran la estructura de riesgos de la movilidad. Esto es, por qué rutas de inclusión o exclusión pueden dirigirse los individuos en materia de accesibilidad. Como se viene argumentando, que estas rutas se concreten o no dependerá de las oportunidades existentes que, en cierta medida, vienen a responder — adecuada o inadecuadamente, dependiendo del momento histórico- a las estructuras de riesgo prevalentes en una ciudad. Las estructuras de riesgo no serán neutrales a un conjunto de atributos adicionales, tales como el género o el nivel socioeconómico, por lo que también será relevante indagar en la asociación entre este tipo de clivajes y las distintas configuraciones del riesgo en materia de accesibilidad.

3 Incluyendo, por ejemplo, desde formación básica para poder leer las señales elementales del sistema, hasta otra más compleja, como la que permite la lectura de la reglamentación del tránsito. 
Una estructura típica de alto riesgo de movilidad es la constituida por los hogares pobres, sin acceso a motorización privada y que viven en la periferia de la ciudad. Sobre ellos debe enfocarse especialmente el estudio de la vulnerabilidad en cuanto a la capacidad de llegar a los lugares que permitan gozar de las oportunidades y recursos distribuidos en la ciudad. Claro está que las distintas combinaciones de recursos determinarán varias configuraciones de riesgo posible frente a una estructura de oportunidades dada.

\section{El transporte público en el esquema AVEO}

Para la realidad latinoamericana, los ingresos y el tiempo del lado de los recursos, y el transporte público del lado de la estructura de oportunidades, constituyen los componentes centrales y de donde van a provenir probablemente los mayores desajustes y, por ende, vulnerabilidades en materia de accesibilidad. En tal sentido, la oferta de transporte público aparece como crucial, en tanto uno de los rasgos de la población más pobre es justamente su cautividad respecto de este modo (Avellaneda García, 2007; Corporación Andina de Fomento, 2009a; Jaramillo, 1993; UN-Habitat, 2011; Vasconcellos, 2010). En efecto, es prácticamente su única alternativa motorizada de movilidad o, al menos, la que dichos sectores pueden pagar para realizar las actividades que desean o necesitan y cuya localización excede la capacidad de llegar caminando. Buena parte de la atención, entonces, debería estar puesta en identificar ajustes y desajustes entre el transporte público y las estructuras de riesgo de movilidad de esta población.

En el esquema propuesto, las oportunidades provenientes del transporte público se colocan del lado del Estado, por su rol protagónico — que podrá ser asumido o no- en materia de regulación y determinación del diseño del bien que se proveerá. Pero el lugar central del transporte público en la discusión responde a que es el mecanismo motorizado con mayor potencial de desmercantilización de la movilidad, y es allí donde debe centrarse la discusión. Esta noción de desmercantilización o decomodificación (Esping-Andersen, 1990) está vinculada a la capacidad del individuo de acceder al bienestar con independencia de su desempeño en el mercado.

En un escenario de máxima desmercantilización, la capacidad de pago del individuo es prácticamente indiferente respecto de la capacidad de utilizar el sistema. En un escenario de mínima desmercantilización, su capacidad para desplazarse de un punto al otro de la ciudad dependerá de forma prácticamente exclusiva de su capacidad de pago, que a su vez está vinculada al desempeño de los individuos en el mercado laboral o a su capital físico heredado. Suponiendo la situación de un individuo desempleado y carente de ahorros u otros ingresos, en un contexto de muy baja desmercantilización su capacidad de movilidad motorizada es prácticamente nula. De hecho, en este extremo, donde no existen elementos regulatorios de la tarifa, se podría incluso argumentar que no existen oportunidades de movilidad entregadas por el Estado más allá de la infraestructura vial básica. En el escenario opuesto, la "pagabilidad" no sería un dato relevante, en tanto el uso no le deman- 
daría gastos de su bolsillo. Bajo máxima desmercantilización o decomodificación, el financiamiento de la provisión del bien provendría de rentas generales, por lo que elementos como el régimen impositivo, así como las estructuras de subsidio y focalización, se vuelven relevantes.

Por supuesto que estos escenarios extremos no tienen cabida en la realidad, pero sí la tienen escenarios más al centro del continuo, donde un ejemplo típico sería aquel en el que los dispositivos desmercantilizadores operan en el momento en que el individuo no puede acceder a determinados bienes por medio del mercado (básicamente, por subsidios). O que, por ejemplo, tienen una base universalista de tal forma de generar una tarifa más baja para todos los ciudadanos a partir de la utilización de subsidios entregados por el sector público.

En otras palabras, y como en otras políticas sociales, las preguntas que hay que responder en el caso del transporte público son tres: quién paga (cuánto y cómo se financia), quién accede al bien y cuál es la calidad del mismo. Si entre los que pagan hay un peso muy alto de los sectores de más altos ingresos y eso no compromete el acceso de los más pobres, se trata de un sistema cuyo potencial desmercantilizador, y también de redistribución progresiva, es alto. Por tanto, conocer quiénes son beneficiarios de los subsidios y cómo se financian es muy relevante. A modo de ejemplo, un sistema de tarifa única en el que no se cobra al usuario por distancia recorrida tiende a favorecer a los sectores localizados en las periferias y en las zonas más alejadas de las zonas centrales de la ciudad. En efecto, los pasajeros que viajan distancias más cortas pagan una tarifa algo más alta de lo que pagarían si la tarifa fuera discriminada, y a la vez quienes recorren más distancias pagan algo menos de lo que les correspondería. Si efectivamente son los sectores más pobres los que se han ido ubicando en la periferia de nuestras ciudades, este tipo de esquema tendría un sesgo progresivo. Por tanto, la discusión depende menos de la existencia o no de un subsidio, y más de la forma en que este opera y de cuál es justamente su efecto desmercantilizador. De todas maneras, este instrumento aparece como un componente muy relevante en esta materia. A modo de ejemplo, en Hernández (2009) es posible observar que la curva de concentración del subsidio al transporte público en Santiago de Chile (asumiendo que se distribuye de forma equivalente a los viajes realizados por estrato socioeconómico) es progresiva. En efecto, los sectores de bajos ingresos concentrarían una proporción más alta de ese dinero que lo que les correspondería si se distribuyera de acuerdo con el ingreso de sus hogares. Al mismo tiempo, esa curva también muestra que el efecto progresivo no comenzaba a tener lugar en el estrato más bajo de la escala, sino en el que lo seguía. Este dato es un buen ejemplo de que en realidad el subsidio al transporte no llega a beneficiar a estos sectores que no hacen uso del servicio, probablemente porque tampoco pueden pagar la tarifa subsidiada.

Por supuesto que, además del aspecto de financiamiento, es necesario tener en cuenta quién es el que efectivamente se beneficia de lo "producido" por el transporte público y con qué calidad. De nada serviría un sistema gratuito y de excelente calidad pero cuya cobertura territorial fuera mínima, por lo que vastos sectores de 
población quedarían excluidos de beneficiarse de él. De la misma forma, tampoco sería eficaz un sistema muy amplio en cobertura y de muy bajo costo - o incluso gratuito-, pero cuya calidad (velocidad, regularidad, seguridad, etcétera) fuera ínfima.

\section{Reflexiones finales}

El argumento central de este artículo es que el esquema AVEO es adecuado para sintetizar los elementos concernientes a la movilidad y la accesibilidad. A lo largo del trabajo se fueron presentando cuáles son esos elementos que deben ser sintetizados, así como los postulados del enfoque AVEO, para finalizar planteando una propuesta específica para el estudio de la accesibilidad. En la exposición se intentó poner de manifiesto varias de las ventajas de este enfoque. Entre ellas, se destaca el carácter amplio que permite razonar de forma simultánea con el conjunto de los elementos que deberían ser considerados. Más aún, es un esquema que facilita sustancialmente la tarea a quienes desean ir más allá del estudio de la accesibilidad y estudiar el rol que este capital juega en la acumulación de otros activos y la búsqueda del bienestar por parte de los individuos y los hogares. Esta amplitud se ve acompañada por una ductilidad del esquema que permite esa integración y abordaje simultáneo.

Más allá de este argumento, su planteo no se expone de forma cerrada o con pretensiones de exhaustividad. En el estado actual de maduración de este esquema analítico, resulta recomendable adoptarlo como un marco general de análisis, aunque aún restan varias tareas pendientes. En primer lugar, es necesario precisar aún más el lugar y la naturaleza de la accesibilidad en cuanto capital. De la misma forma, el rol de la forma urbana debe ser objeto de una reflexión acerca de su posible desagregación en un conjunto de elementos que podrían ser considerados como recursos u oportunidades.

Por otra parte, el carácter general y abarcador de este esquema, si bien es muy atractivo en términos analíticos, conlleva la presencia de cierta ambigüedad en términos de constructos teóricos y contrastación de hipótesis. Será necesaria, entonces, una sólida acumulación de estudios empíricos para lograr niveles adecuados de especificación y precisión en un conjunto de hipótesis básicas las que, recién en ese punto, podrán revestir alguna aspiración de generalidad en sus conclusiones.

La acumulación en estudios empíricos que adopten este esquema ayudará a operacionalizar - y precisar - cuál es el conjunto de recursos y oportunidades más relevantes en el contexto latinoamericano. De esta forma también será posible avanzar en el estudio configuracional de ellos y su asociación con otras variables, como estrato social y género. OEURE 


\section{Referencias bibliográficas}

Antico, C. (2005). Deslocamentos pendulares na região metropolitana de São Paulo. São Paulo em Perspectiva, 19(4), 110-120. http://dx.doi.org/10.1590/S0102-88392005000400007

Aranha, V. (2005). Mobilidade pendular na metrópole paulista. São Paulo em Perspectiva, 19(4), 96 109. http://dx.doi.org/10.1590/S0102-88392005000400006

Ascher, F. (2005). Ciudades con velocidad y movilidad múltiples: un desafío para los arquitectos, urbanistas y políticos. Revista ARQ, 20, 11-19. doi: 10.4067/S0717-69962005006000002

Avellaneda García, P. (2007). Movilidad, pobreza y exclusión social. Un estudio de caso en la ciudad de Lima. Barcelona: Departamento de Geografía, Universidad Autónoma de Barcelona.

Blumenberg, E. (2004). Beyond the spatial mismatch: Welfare recipients and transportation policy. Journal of Planning Literature, 19(2), 182-205. doi: 10.1177/0885412204269103

Brito, F. \& De Souza, J. (2005). Expansão urbana nas grandes metrópoles, o significado das migrações intrametropolitanas e da mobilidade pendular na reprodução da pobreza. São Paulo em Perspectiva, 19(4), 48-63. http://dx.doi.org/10.1590/S0102-88392005000400003

Caiado, M. C. (2005). Deslocamentos intra-urbanos e estructuração socioespacial na metrópole brasiliense. São Paulo em Perspectiva, 19(4), 64-77.

Cass, N., Shove, E. \& Urry, J. (2005). Social exclusion, mobility and access. The Sociological Review, 53(3), 539-555. http://dx.doi.org/10.1111/j.1467-954X.2005.00565.x

Cebollada, Á. \& Avellaneda, P. (2008). Equidad social en movilidad: Reflexiones en torno a los casos de Barcelona y Lima. X Coloquio Internacional de Geocrítica, Diez años de cambios en el mundo, en la geografía y en las ciencias sociales, 1999-2008. Barcelona, 26-30 de mayo de 2008, Universidad de Barcelona.

Church, A., Frost, M. \& Sullivan, K. (2000). Transport and social exclusion in London. Transport Policy, 7(3), 195-205. http://dx.doi.org/10.1016/S0967-070X(00)00024-X

Corporación Andina de Fomento (2009a). Observatorio de Movilidad Urbana para América Latina. Información para mejores politicas y mejores ciudades. Caracas: Corporación Andina de Fomento (CAF). En http://publicaciones.caf.com/publicacion?id=1150

Corporación Andina de Fomento (2009b). Observatorio Movilidad Urbana: 15 ciudades latinoamericanas. XV Congreso Latinoamericano de Transporte Público y Urbano (CLATPU), Buenos Aires, 31 marzo-3 abril 2009

Currie, G. \& Stanley, J. (2008). Investigating links between social capital and public transport. Transport Reviews, 28(4), 529-547. doi: 10.1080/01441640701817197

Currie, G., Richardson, T., Smyth, P., Vella-Brodrick, D., Hine, J., Lucas, K. (. . .) Stanley, J. (2009). Investigating links between transport disadvantage, social exclusion and well-being in Melbourne - Preliminary results. Transport Policy, 16(3), 97-105. http://dx.doi.org/10.1016/j. tranpol.2009.02.002

Esping-Andersen, G. (1990. The three worlds of welfare capitalism. Princeton, NJ: Princeton University Press.

Flamm, M., Jemelin, C., \& Kaufmann, V. (2008). Travel behaviour adaptation processes during life course transitions. A methodological and empirical study using a person-based GPS tracking system. Losana: Laboratoire de Sociologie Urbaine (LASUR).

Flamm, M. \& Kaufmann, V. (2006). Operationalising the concept of motility: A qualitative study. Mobilities, 1(2), 167-189. doi: 10.1080/17450100600726563 
Gobillon, L., Selod, H. \& Zenou, Y. (2007). The mechanisms of spatial mismatch. Urban Studies, 44(12), 2401-2427. doi: 10.1080/00420980701540937

Gutiérrez, A. (2009a). Movilidad o inmovilidad: ¿Qué es la movilidad?. Aprendiendo a delimitar los deseos. XV Congreso Latinoamericano de Transporte Público y Urbano (CLATPU), Buenos Aires, 31 marzo-3 abril 2009.

Gutiérrez, A. (2009b). Movilidad y acceso: embarazo y salud pública en la periferia de Buenos Aires. XV Congreso Latinoamericano de Transporte Público y Urbano (CLATPU) Buenos Aires, 31 marzo-3 abril 2009.

Hernández, D. (2009). El transporte público como política social: Dimensiones de análisis y tensiones, el ejemplo del Transantiago y sus efectos en una comuna Santiago de Chile. Seminario Internacional Movilidad y Equidad, Santiago, 3 y 4 de setiembre de 2009.

Hernández, D. \& Witter, R. (2011). Entre la ingeniería y la antropología: hacia un sistema de indicadores integrado sobre transporte público y movilidad. Revista Transporte y Territorio (Universidad de Buenos Aires), 4, 29-46. En http://www.rtt.filo.uba.ar/RTT00403029.pdf

Jaramillo, J. (1993). Movilidad urbana y modos de vida de sectores populares. Santiago: Instituto de Estudios Urbanos y Territoriales, Pontificia Universidad Católica de Chile.

Jirón, P. (2008). Unravelling invisible inequalities in the city through urban daily mobility. The case of Santiago de Chile. Swiss Journal of Sociology, 33(1, Special Issue on Space, Mobility and Inequality), 45-68.

Kain, J. (1968). Housing segregation, Negro employment, and metropolitan decentralization. 2uarterly Journal of Economics, 82(2), 175-197. En http://www.jstor.org/stable/1885893

Kain, J. (1992). The spatial mismatch hypothesis: Three decades later. Housing Policy Debate, 3(2), 371-392.

Kaufmann, V. (2002). Re-thinking mobility. Contemporary Sociology. Aldershot (Inglaterra)/Burlington (EE.UU): Ashgate.

Kaufmann, V., Bergman, M. M. \& Dominique, J. (2004). Motility: Mobility as capital. International Journal of Urban and Regional Research, 28(4):745-756. En http://www.pacte.cnrs.fr/ IMG/pdf_articleMotilityIJURR.pdf

Kaufmann, V., Viry, G. \& Widmer, E. (2009). Motility. En B. Collet \& N. Schneider (Eds.), Mobile living across Europe. Vol. II de Causes and consequences of job-related spatial mobility in crossnational perspective. Leverkusen-Opladen: Barbara Budrich Publishers.

Kaztman, R.(Coord.). (1999). Activos y estructuras de oportunidades. Estudios sobre las raices de la vulnerabilidad social en Uruguay. Montevideo: Programa de las Naciones Unidas para el Desarrollo (PNUD Uruguay)/Comisión Económica para América Latina y el Caribe (Cepal, Oficina de Montevideo). En http://www.eclac.cl/cgi-bin/getProd.asp?xml=/publicaciones/xml/2/10772/P10772.xml\&xsl=/uruguay/tpl/p9f.xsl

Kaztman, R. (2008). La calidad de las relaciones sociales en las grandes ciudades de América Latina: viejos y nuevos determinantes [Mimeo]. Montevideo.

Kaztman, R. (2009). Territorio y empleo: circuitos de realimentación de las desigualdades en Montevideo. Rethinking Inequalities. XXVIII International Congress of the Latin American Studies Association (LASA), Río de Janeiro, marzo 2009.

Kaztman, R. \& Filgueira, F. (2006). Las normas como bien público y como bien privado: reflexiones en las fronteras del enfoque AVEO. Documentos de Trabajo. Serie Aportes Conceptuales 4. Mon- 
tevideo: Programa de Investigación sobre Pobreza e Integración Social (IPES), Universidad Católica del Uruguay.

Larsen, J., Urry, J. \& Axhausen, K. (2006). Social networks and future mobilities. Lancaster: Lancaster University, Institute for Transport Planning and Systems/Swiss Federal Institute of Technology, Zurich. Report to the UK Department for Transport.

Lazo, A. (2008). Transporte, movilidad y exclusión. El caso de Transantiago en Chile (Coloquio Internacional de Geocrítica, 10², 2008, Barcelona). Scripta Nova, Revista Electrónica de Geografia y Ciencias Sociales, 12(270). En http://www.ub.edu/geocrit/sn/sn-270/sn-270-45.htm

Massot, M.-H. \& Orfeuil, J.-P. (2005). La mobilite au quotidien, entre choix individuel et production sociale. Cahiers Internationaux de sociologie, 1(118), 81-100.

Miralles-Guasch, C. (2006). Usos del tiempo y movilidad. Barcelona: Ajuntament de Barcelona.

Miralles-Guasch, C. \& Cebollada, A. (2003). Movilidad y transporte. Opciones politicas para la ciudad. Madrid. Fundación Alternativas.

Moser, C. (1998). The asset vulnerability framework: Reassessing urban poverty reduction strategies. World Development 26(1), 1-19. http://dx.doi.org/10.1016/S0305-750X(97)10015-8

Neutens, T., Delafontaine, M. \& Schwanen, T. (2010). Social equity, accessibility and the temporal dimension of public service delivery. XII World Conference on Transportation Research (WCTR), 11 al 15 de julio de 2010, Lisboa.

Rodríguez Vignoli, J. (2008). Movilidad cotidiana, desigualdad social y segregación residencial en cuatro metrópolis de América Latina. EURE, 34(103), 49-71. En http://www.eure.cl/wpcontent/uploads/2008/12/EURE_103_03_RODRIGUEZ.pdf

Social Exclusion Unit. (2003). Making the connections: Final report on transport and social exclusion. Londres: Autor. En http://assets.dft.gov.uk/statistics/series/accessibility/making-theconnections.pdf

Titheridge, Helena. (2006). Social exclusion and transport policy [mimeo]. Londres, University College.

UN-Habitat (United Nations Human Settlements Programme). (2011). UN-Habitat Series on Regional Trends and Initiatives in Sustainable Urban Mobility. Vol. 1: Latin America and the Caribbean. Nairobi: Autor.

Urry, J. (2007). Mobilities. Cambridge \& Malden: Polity Press.

Vasconcellos, E. A. (2010). Análisis de la movilidad urbana. Espacio, medio ambiente y equidad. Bogotá: Corporación Andina de Fomento (CAF).

Viry, G., Kaufmann, V. \& Widmer, E. (2009). Social integration faced with commuting: More widespread and less dense support networks. En T. Ohnmacht, H. Maksim \& M. M. Bergman, Mobilities and inequality (pp. 121-143). Aldershot: Ashgate.

Wenglenski, S. (2003). Une mesure des disparites sociales d'accesibilite au marche de l'emploi en Ile-De-France. Val de Marne, París: Centre de Recherche sur l'Espace, les Transports, l'Environnement et les Institutions Locales, Institut d'urbanisme de Paris, Université de Paris XII.

Wenglenski, S. (2006). Regards sur la mobilité au travail des classes populaires. Una exploration du cas parisien. Les Cabiers Scienti fiques du Transport, 49, 103-127.

Wenglenski, S. \& Orfeuil, J.-P. (2006). The differences in the accesibility to the job market according to the social status and the place of residence in the Paris Area [mimeo]. 